\title{
A Survey: Issues and Challenges in Vehicular Adhoc Network (VANET)
}

\author{
Patel Suhel $^{1}$, Shaikh Sharique Ahmad ${ }^{2}$, Pathan Bilalkhan ${ }^{3}$, Mohammad Asif $^{4}$, Patil \\ Harshal S. ${ }^{5}$ \\ 1,2,3,4,5 Department of Computer Engineering, Jamia Institute of Engineering and Management Studies, Akkalkuwa
}

Published on: 12 September, 2021

\begin{abstract}
Wireless technology is advancing rapidly with time. The VANET (Vehicle Adhoc Network, i.e., V2V (Vehicle to Vehicle) communication) concept is the most growing research work area in the field of wireless communication. With the huge advancement in the VANET, there is a great revolution in the field of wireless communication. We can achieve the services in terms of network availability, fast handovers and security in advanced applications. With the passage of time the VANET technology is advancing. To make the network more strong and efficient there are many issues that has to be addressed. Various research works related to the applications, protocols and security in VANET are studied and discussed in this article. We can implement crash warning applications, to enables roles in future road safety in VANET with short communication latency and not needing an infrastructure. But in VANET there are some additional security challenges and difficulties to detect and exclude vehicles. That is malicious nodes from the network. In VANET the nodes are communicating with each other directly and not through a base station or a server which causes the issue besides wireless communication's which inherent security vulnerabilities, such as eavesdropping, jamming, and tampering. In view of aforesaid, in this article we have mentioned and discussed the detail study of various research work related to the Security and implementation issues in VANET. Moreover, after reviewing the existing works, we have analyzed them and found the pros and cons for our future research.
\end{abstract}

Keywords: Vehicular area network, communication latency, jamming and eavesdropping vulnerabilities

\section{I -INTRODUCTION}

T he term VANET is acronym for vehicular ad-hoc networks was originally adopted to reflect the ad-hoc nature of the highly dynamic networks. First, consider the opportunities. If the vehicles can directly interact altogether and with infrastructure, a new paradigm for vehicle safety applications can be created. Second, further challenges are created by high vehicle speed and highly dynamic operating environments. Third, new requirements include new expectations for high packet delivery rates and low packet latency. Further, customer acceptance and governmental oversight bring very high expectations of privacy and security. Driving means constantly changing location. A very important category is driver assistance and car safety[1]. Another category is infotainment for passengers. VANET communication is based on two types. [2] Vehicle-to-Vehicle (V2V) communication, (2) Vehicle-to-Infrastructure (V2I) communication. In $\mathrm{V} 2 \mathrm{~V}$ communication, VANET communication can be donedirectly between vehiclesas-one- hop\| communication, such as car-to-car communication. In V2Icommunication, VANET 


\section{International Journal of Innovations in Engineering and Science, www.ijies.net}

communication can be done between vehicles and road side infrastructure as-multi- hop\| communication. In this paper, we examine the possibility of deploying a SelfAdaptive Prioritized Traffic Signal Controller in VANET, which receives information from vehicles, such as the vehicle's location i.e. its real position by GPS and its speed, and then use this information to optimize the traffic signal scheduling at the intersection

To control traffic signals there are so many technologies were proposed. In the management of traffic the Emergency Vehicles is highly considerable fact to avoid time delays in the services of EV. Recently increasing the number of cars on city roads has There are many problems created, such as traffic density, people get killed in accidents, emissions, fuel consumption, etc. Emergency vehicle such as ambulances, fire trucks, and police vans are special vehicle designated to respond to emergency immediately. Thus, reaching their destination as fast as possible is their primary concern. Due to traffic congestion, response time of emergency vehicles is increases. Emergency vehicles should be able to respond to emergency calls for an incident with minimum delay. Where time delays in the services of EV causes human lives risks as well as financial losses. Therefore, need a Traffic Management System that responsible for managing traffic with emergency vehicle Consideration, on the roads, efficiently. That can be possible by applying the priority preferences to vehicles. So we motive and avoid this problem by prioritizing emergency vehicles at the traffic signal intersection, Where EV having higher priority than other vehicles. Exists work manages and controls the traffic using the VANET technology, and focuses only on job scheduling, where jobs are the Platoon (the Platoons are the group of one or more vehicles that are to be cross through the intersections) [1]. But the priority considerations for Emergency Vehicles are not considered in this System. This makes the more chances of time delays in the services of EV. Hence in exists work only applicable to reduce conflicts at intersections but not to overcome the time delays of EV. This work focuses on the particular problem of traffic management for emergency services, for which a delay of few Minutes in the services of EV may cause human lives risks as well as financial losses. In our proposed scenarios the idea to solve the problem with traffic emergency control, by designing Emergency Vehicle Priority Preference at Intelligent Road Traffic Signal
Control System in VANET where the emergency vehicles will be consider first based on calculated arrival time at the intersections. Every Emergency Vehicle is always deals with high priority than other vehicles. The Priority Scheduling Algorithm schedules the higher priority vehicles and provides the circumstances in such a way that, the EV will scheduled to high priority vehicles and cross over intersection without any conflict at intersection [1].

\section{II- LITERATUREREVIEW}

Vehicle-to-vehicle communication is a very actual and challenging topic. Vehicles equipped with devices capable of short-range wireless connectivity can form a particular mobile ad-hoc network, VANET_-Vehicular Ad-hoc Network. The existence of such networks opens the way for a wide range of applications. Two of most important classes of such applications are those related to traffic safety and route planning. Route planning aims to provide drivers with real-time traffic information, which, in the absence of a VANET, would require an expensive infrastructure. By contrast, the VANET approach is scalable and has low maintenance costs. Moreover, shortrange wireless communication technologies have no associated cost, other than the communication devices. Safety applications involve disseminating urgent information, which is not present in the driver's field of view, or it is difficult to notice for reasons such as fog or other vehicles obstructing the line of sight. For instance, a lot of accidents occur in foggy conditions because drivers notice too late that some kind of incident has occurred in front of them. Safety at intersections could also be enhanced, since the risk of collisions could be detected in advance and the driver could be warned seconds before what would otherwise be an imminent accident. The evaluation of VANET protocols and applications could be made through real outdoor experiments, which are timecostly and claim for a large number of resources in order to obtain significant results. Instead, simulation is a much cheaper and easier to use method. Obviously, this leads network and application developers to use simulation in order to evaluate different simple or complicated and innovative solutions before implementing them.

A. Vehicular Ad hoc Network(VANET) 


\section{International Journal of Innovations in Engineering and Science, www.ijies.net}

The Vehicular ad hoc networks (VANETs) are basically using the working principles of mobile ad hoc networks (MANETs), where MANET is the spontaneous creation of a wireless network for data exchange to the domain of mobiles [6]. In 2001 VANETs were first mentioned and introduced under "car-to-car ad hoc mobile communication and networking" applications, where networks for vehicles can be formed and information can be relayed among cars. There is vehicleto-vehicle and vehicle-to- roadside communications architectures will co-exist in VANETs to provide road safety, navigation, and other roadside services.

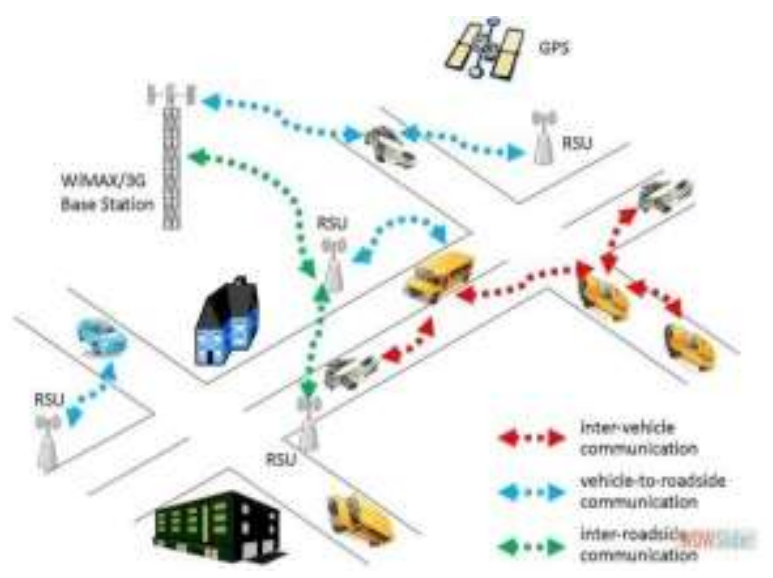

Fig 1- Overview of VANET VANETs

are sometimes referred as Intelligent Transportation Networks because it is the key part for the transportation systems framework. In the early 2000s, VANETs were seen as only one-to-one application of principles of MANET. The more generic term inter-vehicle communication (IVC) was changes to the mostlysynonymoustermVANETupto2015

[5], but the focus remains on the aspect of spontaneous networking and less focus on the use of road side infrastructures like Road Side Units (RSUs).

\section{B. Communication in VANET}

In a typical VANET, Communication takes place in between on-board unit (OBU), installed at vehicle and there are road-side units (RSU) installed along the roads. In backend, the trusted authority (TA) or some other application servers are installed for additional support.
The Dedicated Short Range Communications (DSRC) is used for interaction of OBUs with RSUs over the wireless channel [10]. But the backend application servers or TA communicate with RSUs using a secure fixed network

(e.g. the Internet). VANET communication is based on two types. [2] Vehicle-to-Vehicle (V2V) communication, (2) Vehicle-to-Infrastructure (V2I) communication. In $\mathrm{V} 2 \mathrm{~V}$ communication, VANET communication can be done directly between vehiclesas-one- hop\| communication, such as car-to-car communication. In V2I communication, VANET communication can be done between vehicles and roadside infrastructure as-multi-hop communication. The onboard sensors invehicles are deals with this mechanism and standard wireless communication protocols specifically for vehicular applications for communication, vehicles can use wireless communications for vehicle-to-vehicle (V2V) orvehicleto-infrastructure (V2I) communications, as described in the dedicated short-range communications/wireless access in vehicular environments standards operating in the spectral range of 5.85- 5.95 GHz [4]. For example, all vehicles are already equipped with a speed sensor or meter. In addition, new vehicles are fortunately being equipped with Global Positioning System (GPS) units that can provide the information of location [5]. As such, a VANET can also be interpreted as a sensor network because the traffic control center or some other central servers can collect lots of useful information about road conditions from vehicles. It is natural to investigate how to utilize the collected real time road conditions to provide useful applications.

The combination of both short-range wireless technology and ad-hoc networking facilitates $\mathrm{C} 2 \mathrm{C}$ and $\mathrm{C} 2 \mathrm{I}$ communications, known as Car-to-X (C2X) communications [9]. Usually viewed as a critical element of the Intelligent Transport Systems(ITS) architecture, VANETs provide solutions for improving road safety and to enable a set of onboard potential services for drivers and passengers as well as different communication facilities between moving vehicles. The importance and potential impact of VANETs have been confirmed by the considerable attention given from the research community and the automotive industry as well as from Governmental authorities and standardization organizations. 


\section{International Journal of Innovations in Engineering and Science, www.ijies.net}

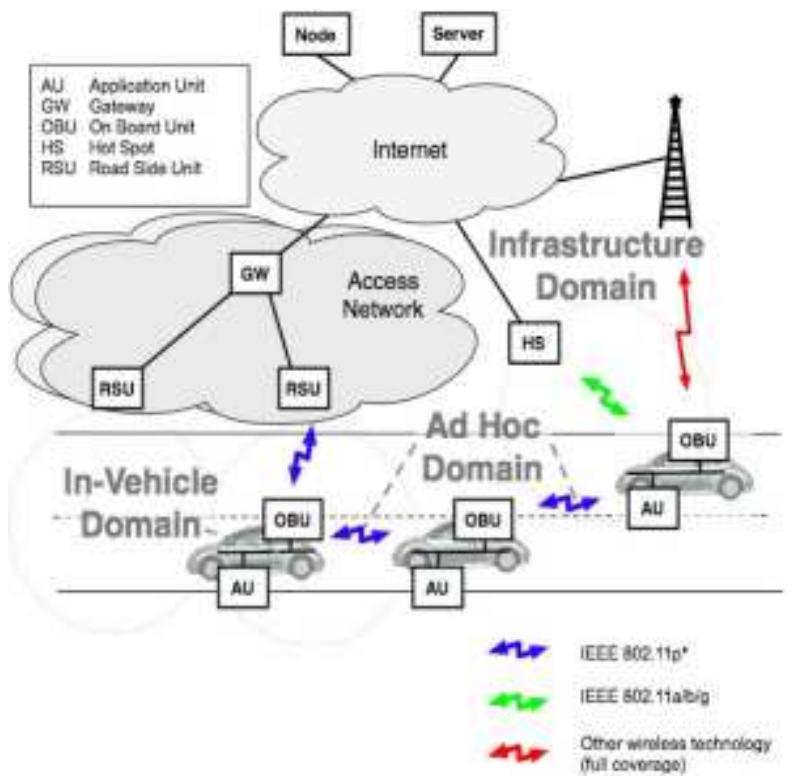

Fig.2-Car-to-X (C2X) communications

The In-Vehicle Domain refers to the vehicle internal network. Each vehicle is equipped with an On-Board Unit (OBU) that implements the communication protocol stack and offers an interface to driver and passenger devices, called Application Units (AUs).

The Ad-hoc Domain is composed of vehicles equipped with OBUs and fixed network nodes installed along roads, Road- Side Units (RSUs). OBUs and RSUs form a VANET, which allows communications in a fully distributed and self-organized manner, without the need of centralized coordination. OBUs directly communicate if wireless connectivity exists among them, or perform multi-hop communications through the use of a dedicated routing protocol.

Finally, in the Infrastructure Domain, the RSU can simply extend the VANET coverage by acting as forward ingenuity, or be attached to an infrastructure network, which in turn can be connected to the internet. The RSU will allow the OBUs to access such infrastructure, and hence, the vehicle AUs can communicate with any host on the internet.

The C2C-CC basic system is composed by individual components:

An Application Unit (AU) is a dedicated entity that runs applications and uses the OBUs communication capabilities. An AU can be embedded in the vehicle and be permanently connected to an OBU, or can be dynamically plugged into the in-vehicle network by drivers or passengers. It can also be a portable device such as laptop or PDA. Multiple AUs are allowed to a single OBU.

An On-Board Unit (OBU) implements the communication protocol stack and provides $\mathrm{C} 2 \mathrm{X}$ communications services to AU's. OBUs are equipped with at least one network device for short-range wireless communications based on IEEE 802.11p, and may also be equipped with more network devices from different technologies. Its main functions and procedures include wireless radio access, geographical ad-hoc routing, network congestion control, IP mobility support, and others.

The Road-Side Units (RSUs) are fixed nodes placed along the roads or highways, or at dedicated locations such as traffic signs, parking places or gas stations. The RSU has the same communication capabilities of OBUs. Is equipped with at least one device for short-range wireless communications based on IEEE 802.11p, and may also be equipped with other network technologies in order to allow communications with an infrastructure network. RSUs may extend the communication range of the Ad- hoc Domain, may provide internet connectivity or may cooperate with other RSUs in forwarding information.

\section{VANET Applications}

In real time message information dissemination of different type, VANETs support the different types of applications - from simple one hop information dissemination to multi-hop dissemination of messages over large distances. Where, simple one hop information dissemination is Cooperative awareness messages [8]. Some of working phenomenon of MANET's are as VANET, but details are different. In MANET, mobiles are moving at random axis, but vehicles are tends to move in an organized way, and most of the vehicles are restricted to move in the specific range, for example, The vehicle range of motion is limited. Following are some range of applications for VANET [7]:

- $\quad$ Electronic brake lights: Brake event information is spread to others, it is useful for other drivers to react to vehicles braking in case of obscured views, which caused by fog on road due to environmental changes.

- Platooning: Platoons are group of vehicles, which are moving on road very closely altogether by following to the leading vehicle through wirelessly 


\section{International Journal of Innovations in Engineering and Science, www.ijies.net}

receiving speed acceleration and steering rotavation information, the moving vehicles forms the road trains by following leader rules.

- Traffic information systems: The vehicles on the road are with electronic information, such as speed, GPS location, etc. This can be providing by communication in VANET to deal with traffic, like as intelligent traffic control system.

- Road Transportation Emergency Services: In daily transportation services, emergencies are even important to handle. Reduce in time delays and speed up of emergency rescue operations are possible by VANET communications, where VANET networks, and road safety warning and status information dissemination are used.

- On-The-Road Services: It is also envisioned that future transportation highway would be one that is "information-driven" and "wirelessly- enabled". While a driver drives on the road, he can discover services (shops, gas stations, etc) on that street by using VANET and even if ongoing sale can be notified at that moment.

\section{About Traffic Management}

The Traffic Management System manages the available traffic passing on the roads well and efficiently. It is very important and necessary to improve this system, because of rapidly change in the volume of traffic with respect to time.

\section{E $\quad$ VANET Enabled in traffic signs}

Understanding traffic sign information correctly is crucial. It helps the driver to anticipate future situations, make decisions and respond in an appropriate way. There are many different kinds of road signs and they are mostly placed above or beside highways and streets. However, these traditional static traffic signs have known limitations. The period of time the drivers have to analyze the information is limited, and even if the road signaling is predominantly standardized, most of the signs use text to convoy meaning restricting the understanding to readers of the language. They are likely to be overlooked during complex driving tasks, and sometimes, the adverse weather conditions or vehicles blocking the line-of-sight between the driver and the sign, make its recognition very difficult. In contrast to traditional traffic signs, traffic signs displayed within the vehicle will solve a big amount of these limitations, as well as they will provide additional help to the driver on his driving tasks. In fact, in-vehicle traffic signs are one of the main ITS technologies. Expensive systems, such as interactive Variavel Message Signs (VMSs)

[17] can present traffic-related information and guidance to drivers through electronic signs located beside or above the highway. The main advantage of these systems is that they can present real-time information that cannot be displayed on traditional static traffic signs, such as information related to hazards, traff

ic conditions, parking, public transport or environment. A special category of VMS, the called Vehicle-Activated Signs (VASs) [17] has been designed to deliver targeted warning information to individual drivers when they exceed a particular vehicle performance threshold (usually speed or following distance). Road sensors, such as buried induction loops or microwave detector heads have been used to monitor individual vehicle behaviors. Unlike the traditional road sign, in which the communication takes place between the sign and the driver, the behavior of the driver can influence the information is displayed on the VMS.

\section{CHALLENGING ISSUE INVANET}

The technical challenges deals with the technical obstacles which should be resolved before the deployment of VANET. Some challenges are given below:[2] Network Management: Due to high mobility, the network topology and channel condition change rapidly. Due to this, we can't use structures like tree because these structures can't be set up and maintained as rapidly as the topology changed.

- Congestion and collision Control: The unbounded network size also creates a challenge. The traffic load is low in rural areas and night in even urban areas. Due to this, the network partitions frequently occurs while in rush hours the traffic load is very high and hence network is congested and collision occurs in the network.

- Environmental Impact: VANETs use the electromagnetic waves for communication. These waves are affected by the environment. Hence to deploy the VANET the environmental impact must be considered.

- MAC Design: VANET generally use the shared 


\section{International Journal of Innovations in Engineering and Science, www.ijies.net}

medium to communicate hence the MAC design is the key issue. Many approaches have been given like TDMA, SDMA, and CSMA etc. IEEE 802.11 adopted the CSMA based Mac for VANET.

- Security: As VANET provides the road safety applications which are life critical therefore security of these messages must be satisfied.

\section{F. SECURITY ISSUES INVANET}

Among all the challenges of the VANET, security got less attention so far. VANET packets contains life critical information hence it is necessary to make sure that these packets are not inserted or modified by the attacker; likewise the liability of drivers should also be established that they inform the traffic environment correctly and within time. These security problems do not similar to general communication network. The size of network, mobility, geographic relevancy etc makes the implementation difficult and distinct from other network security[2].

\section{G. Attacks in the VANET}

To get better protection from attackers we must have the knowledge about the attacks in VANET against security requirements. Attacks on different security requirement are given below[2]: Impersonate: In impersonate attack attacker assumes the identity and privileges of an authorized node, either to make use of network resources that may not be available to it under normal circumstances, or to disrupt the normal functioning of the network. This type of attack is performed by active attackers. They may be insider or outsiders. This attack is multilayer attack means attacker can exploit either network layer, application layer or transport layer vulnerability. This attack can be performed in two ways: a) False attribute possession: In this scheme an attacker steals some property of legitimate user and later with the use of attribute claims that it is who (legitimate user) that sent this message. By using this type attack a normal vehicle can claim that he/she is a police or fire protector to free the traffic. b) Sybil: In this type of attack, an attacker use different identities at the same time.

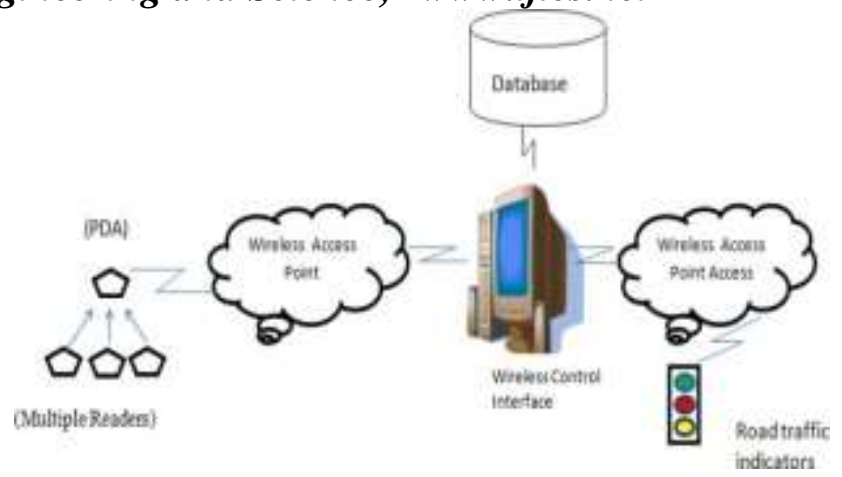

Figure 1.3: A frame work for Intelligent Road Traffic Signal Control System Using Multiple Wireless Network Sensors (MWNS)

\section{III -CONCLUSION}

In this survey we realized that for VANET implementation Security is the major issue. We studied the security requirements and challenges to for VANET and security measure in this article. Also discussed different types of attacks and their solutions for VANET. We discuss some technologies which are used in the different solutions. As we studies the privacy is the major issues in VANET. However confidentiality is not required in the VANET because generally packets on the network do not contain any confidential data.

\section{REFERENCES}

[1] Vanet Based Selfadaptive Prioritized Traffic Signal Controll, Shaikh Sharique Ahmad, International Journal of Innovative Research in Computer and Communication Engineering,Vol. 6, Issue 4, April2018

[2] Security Challenges, Issues And Their Solutions For Vanet\| by Ram Shringar Raw, International Journal of Network Security \& Its Applications (IJNSA), Vol.5, No.5, September 2013.

[3] Adaptive Traffic Signal Control With Vehicular Ad hoc Networks $\|$ by Kartik Pandit, Dipak Ghosal, Member, IEEE, H. Michael Zhang, and Chen-Nee Chuah, Senior Member, IEEE, IEEE TRANSACTIONS ON VEHICULAR TECHNOLOGY, VOL. 62, NO. 4, MAY2013.

[4] Ieee802.11p: Towards an international standard for wireless access in vehicular environments, $\|$ by $D$. Jiang and L. Delgrossi, in Proc. IEEE VTC Spring, May 2008, pp. 2036-2040.

[5] C. Priemerand B. Friedrich, $-A$ decentralized adaptive traffic signal control using v2I 
Vol. 6 , No. 10, 2021, PP. 238-244

International Journal of Innovations in Engineering and Science, www.ijies.net communication data,\| in Proc. 12th Int. IEEE ITSC, Oct. 2009, pp.1-6.

[6] Morteza MohammadiZanjireh; HadiLarijani (May 2015). A Survey on Centralised and Distributed Clustering Routing Algorithms for WSNs,IEEE 81st VehicularTechnologyConference.Glasgow, Scotland. doi:10.1109/VTCSpring.2015.71456 50.

[7] Sommer, Christoph; Dressler, Falko (December 2014). Vehicular Networking. Cambridge University Press.ISBN 9781107046719.

[8] "A Comparative study of MANET and VANET Environment". Journal of Computing. 2 (7). July 2010. Retrieved 28 October2013.

[9] F. Wang, D. Zeng, andL. Yang, -Smart Cars on Smart Roads: an IEEE Intelligent Transportation Systems Society Update,\| IEEE Pervasive Computing, Vol. 5, No. 4, pp.68-69, 2006.

[10] H.Oh,C.Yae,D.Ahn,andH.Cho,-5.8 GHz DSRC Packet Communication System for ITS Services, $\|$ in Proceedings of the IEEE VTC '99, Sept. 1999, pp. 2223 $-2227$. 